\title{
Les impasses de l'école multiculturelle et de l'école républicaine dans une perspective comparative
}

\begin{abstract}
Abdeljalil Akkari
Ce texte analyse dans une perspective comparative le défi que représente l'accroissement de la diversité culturelle dans les systèmes éducatifs des pays industrialisés. Dans la première partie du texte, nous reviendrons sur le contentieux historique entre forme scolaire et certains groupes sociaux. La deuxième partie s'attache à analyser la persistance du traitement inégal des minorités ethniques dans différents système scolaires. La troisième partie propose la notion de minorité postcoloniale comme un enrichissement de l'approche utilisée par l'anthropologue John Ogbu dans son analyse des trajectoires scolaires des jeunes minoritaires. Nous aborderons dans la dernière partie la perspective de recherches futures permettant à la forme scolaire de devenir plus sensible aux différences culturelles.
\end{abstract}

\section{Introduction}

Au-delà des politiques éducatives spécifiques mises en œuvre dans chaque contexte national, les recherches effectuées aux Etats-Unis, en France ou en Suisse montrent les difficultés et les défis de la gestion de l'hétérogénéité culturelle liée aux migrations internationales et à la reconnaissance des minorités ethniques ${ }^{1}$. Dans ces trois contextes l'école publique se montre incapable de promouvoir une véritable intégration des populations socio-culturellement distantes de la norme scolaire, notamment des migrants, en respectant et en valorisant leurs identités culturelles. Dans la première partie du texte, nous reviendrons sur le contentieux historique entre forme scolaire et certains groupes sociaux. La deuxième partie s'attache à analyser la persistance du traitement inégal des minorités ethniques dans différents systèmes scolaires. La troisième partie propose la notion de minorité postcoloniale comme un enrichissement de l'approche des trajectoires scolaires des jeunes minoritaires utilisée par John Ogbu. À partir de ces constats, nous esquisserons les recherches à notre avis nécessaires pour fournir des pistes permettant à la forme scolaire de devenir plus sensible aux différences culturelles, notamment en termes de formation initiale et continue des enseignants. 


\section{Massification de la forme scolaire et cultures}

Un retour historique sur le développement de la forme scolaire est nécessaire pour comprendre la nature des tensions actuelles entre forme scolaire et cultures d'élèves considérées comme éloignées de la norme. En Europe, un lien étroit entre religion et école a subsisté jusqu'au 18ème siècle, où on a assisté à l'éviction progressive de l'Église comme acteur quasi unique de la scène scolaire. La philosophie des Lumières, alliée au développement de l'État-Nation propose la raison comme alternative fondant l'action citoyenne (Ruano-Borbalan, 2002). La scolarisation comme phénomène institutionnel apparaît ainsi dans le monde contemporain comme le bras long de l'État-Nation.

La massification de la forme scolaire en Europe s'est réalisée progressivement tout au long de la deuxième moitié du 19ème siècle. Cette forme scolaire désigne un nouveau processus d'institution par incorporation de règles sociales dans le fonctionnement pédagogique (Vincent, 1994). La désignation en français des enseignants par le terme «instituteurs» rappelle bien ce processus. Du point de vue pédagogique, la forme scolaire possède plusieurs caractéristiques. L'enseignement s'adresse au groupe classe et la scolarité est organisée en degrés successifs. Le statut particulier de la culture de l'écrit, l'apparition des disciplines scolaires et des institutions de formation des enseignants sont également spécifiques au rapport pédagogique institué par la forme scolaire. L'existence d'une forme scolaire marque des inclusions, des exclusions et des équilibres nouveaux entre école comme institution et société en voie d'industrialisation; entre scolarisés et nonscolarisés, entre ceux qui fréquentent durablement la forme scolaire et ceux qui en sortent d'une manière précoce. En bref, l'institution scolaire moderne, instaurée par la promulgation de l'obligation scolaire se caractérise par quatre paramètres principaux. Premièrement, elle est largement liée au développement de l'appareil étatique et de l'industrialisation. Autrement dit, le système éducatif apparaît comme un pilier du nouveau rapport entre individu et pouvoirs politique et économique. Deuxièmement, le système éducatif a une vocation universelle, c'est à dire qu'il est destiné d'une part à tous les enfants quelle que soit leur origine sociale, d'autre part, il est le garant d'un savoir scientifique qui se veut universel et rationnel. Troisièmement, l'apparition de l'école coïncide avec l'émergence de l'enfance et ensuite de l'adolescence comme périodes spécifiques de la vie d'un individu. Quatrièmement, l'essor des systèmes éducatifs européens est intimement lié à la formation des états modernes, la plupart du temps monolingues. L'ensemble de ces quatre paramètres nous renvoie, en somme, à une institution scolaire ambiguë ou ambivalente. D'une certaine façon, elle unit les futurs citoyens par la fréquentation de mêmes lieux et les met durablement en contact avec les mêmes savoirs scientifiques et scolaires pendant au moins la scolarité obligatoire. Elle leur ouvre un nouveau monde en dehors de leurs sphères familiale et sociale. Mais en même temps, elle les divise, puisqu'elle est au service d'une nation, fondée sur une culture et habituellement une langue, à l'exclusion 
des autres présentes sur son territoire. Il s'agit là d'un double phénomène d'homogénéisation au sein d'une culture dominante (dite nationale) et de relégation des cultures minoritaires.

Historiquement, la forme scolaire a un contentieux culturel avec trois groupes sociaux bien identifiés: les populations rurales, les classes sociales laborieuses et les minorités linguistiques et ethniques. Ce contentieux est lié à la discontinuité culturelle entre les pratiques, postures et orientations valorisées par ces groupes et celles dominantes au sein de la forme scolaire. Ainsi, les populations rurales se rendent rapidement compte du caractère extractif de l'école, dans la mesure où les élèves ruraux réussissant leur scolarité finissent par quitter les régions rurales, les vidant ainsi de leur élite. Les classes ouvrières de la fin du 19ème siècle privilégient, elles, la solidarité et une proximité immédiate avec le travail productif. Or, l'école valorise à la fois la compétition entre élèves et la séparation entre activité intellectuelle et travail manuel. En ce qui concerne les minorités linguistiques et ethniques, l'irruption de la forme scolaire a accéléré leur assimilation linguistique tout en maintenant leur marginalisation politique. Conjuguée avec développement de l'industrialisation, de l'urbanisation et avec l'élimination des petites exploitations agricoles, la pénétration de l'école comme institution a contribué à l'exacerbation des conflits internes dans ces trois groupes. Exode rural pour le premier, mobilité sociale inachevée pour le second et bilinguisme et biculturalisme de transition avec ses possibles conflits identitaires pour le troisième. Il nous semble impossible de comprendre les difficultés actuelles de gestion des différences culturelles dans les systèmes éducatifs européens sans les situer dans le cadre de ce contentieux historique que nous venons de décrire. Les modèles républicain et multiculturel analysés dans la partie suivante de cet article ont tenté de trouver des solutions à ce contentieux historique.

\section{Diversité des modèles de gestion des différences à l'école et persistance des inégalités}

On oppose fréquemment le modèle républicain à la française au modèle multiculturel d'inspiration anglo-saxonne à propos de la gestion des différences culturelles à l'école (Jamet, Lenoir \& Xypas, 2006; Kintzler, 1998). Au delà des contextes historiques particuliers et des différences entre ces deux modèles, nous tenterons de démontrer qu'ils aboutissent aux mêmes impasses actuelles en ce qui concerne la scolarisation des minorités ethniques. Cette dernière notion renvoie dans cet article à deux dimensions principales. D’une part, aux représentations dominantes des acteurs scolaires (enseignants, élèves, parents, administrateurs, chercheurs, etc.) à propos d'une partie des élèves en tant que population scolaire spécifique, problématique et différente à cause de ses origines ethniques, culturelles ou linguistiques. D'autre part, cette notion renvoie aux constats fac- 
tuels mettant en évidence une «discrimination avérée» ou des «inégalités de traitement» de cette même population au sein de l'espace scolaire.

\section{Le modèle républicain à L'épreuve des banlieues}

La France apparaît comme l'archétype du modèle républicain universel de la gestion des différences à l'école. Le modèle républicain ${ }^{2}$ se base sur l'engagement sans faille des enseignants au service de la République. Ce modèle a géré les différences à travers trois orientations principales: le mérite, l'indifférence aux différences et la laïcité. Le mérite exprime une orientation récompensant ceux qui réussissent le mieux à l'école et dans la société. Il a fallu attendre les travaux des sociologues de l'éducation des années 60 pour se rendre compte que le mérite est un instrument utilisé par les classes dominantes afin de légitimer la reproduction des inégalités sociales par l'école (Bourdieu \& Passeron, 1970). L'indifférence aux différences est notamment illustrée par le refus de trop régionaliser les programmes scolaires et l'interdiction des signes religieux ostensibles. C'est donc, une véritable assimilation des élèves aux valeurs communes de la République et une disparition des particularismes qui sont recherchées par le modèle scolaire républicain. La laïcité se veut une rupture avec la présence puissante de l'Église catholique au sein des institutions éducatives françaises. À ce propos, il faut rappeler que le maintien dans le pays d'un important secteur d'enseignement catholique $^{3}$, bénéficiant de subventions publiques, montre bien que la laïcité peut être à géométrie variable. Elle peut s'accommoder avec les particularités historiques et les rapports entre les forces politiques. Le laïcisme érigé en dogme peut cependant bloquer la connaissance nécessaire du fait religieux par les enseignants et les élèves. Or, on sait que l'étude, dès le plus jeune âge, des pratiques culturelles des différentes communautés religieuses présentes à l'école peut favoriser l'ouverture d'esprit, ce qui souligne la nécessité, malgré les difficultés, d'enseigner le fait religieux (Benbassa \& Attias, 2007).

Les minorités ethniques en France ont été d'abord animées par la double revendication de pouvoir accéder aux niveaux supérieurs de scolarité et à une insertion économique. Même si les hommes politiques quand ils parlent des banlieues brandissent souvent le spectre du communautarisme, celui-ci n'a pas été à l'origine l'horizon mobilisateur. Ainsi, la marche nationale des jeunes d'origine maghrébine il y a plus de 20 ans avait fait apparaître au grand jour une frange de la population que l'on peut appeler «minorité ethnique» et qui exigeait l'inclusion dans la société française et la fin des discriminations. Malgré ces revendications, la situation des jeunes d'origine maghrébine n'a cessé de se détériorer durant les dernières décennies. Tous les observateurs s'accordent à dire que la ségrégation scolaire est concomitante de la ségrégation urbaine. L'école publique fonctionne en vase clos et constitue à la limite un corps étranger dans certaines banlieues. Pour qualifier ce phénomène, Maurin (2004) utilise le terme de ghettoïsation dans la mesure où l'on assiste depuis de nombreuses années à un séparatisme territorial, amenant les classes moyennes supérieures à fuir les professions 
intermédiaires, puis les ouvriers à fuir les chômeurs immigrés. Si la ségrégation urbaine n'atteint pas encore en France les proportions de certaines villes américaines, il n'en demeure pas moins que les deux dernières décennies ont vu l'émergence de ce qu'on appelle les «problèmes des banlieues» et plus récemment celui du "fondamentalisme islamique».

L'école républicaine semble incapable de gérer la diversité ethnique induite par les migrations en provenance des anciennes colonies. Dans l'un de ses derniers travaux de terrain, Bourdieu a pu mettre en lumière la situation d'exilés de l'intérieur que vivent les jeunes marginalisés issus pour la plupart de l'immigration (Bourdieu, 1991, 1993). Il s'agit de jeunes (souvent d'origine maghrébine) dont l'école ne sait que faire et qui sont insérés dans des quartiers difficiles où leur vie est meublée par la débrouille et la petite délinquance. Ces jeunes sont ballottés d'un stage à l'autre dans une scolarité qui se poursuit sans finalité précise et sans possibilité réelle d'insertion professionnelle. Les entretiens réalisés par Bourdieu et son équipe révèlent une profonde crise scolaire et sociale. D'autres travaux montrent qu'à l'instar des Etats-Unis, les élèves maghrébins qui réussissent le mieux à l'école mettent en œuvre des stratégies d'évitement de leurs quartiers et de leurs communautés d'origine (Vincent, 1994). Autrement dit, la réussite scolaire semble rimer avec assimilation républicaine et coupure des ponts culturels avec les communautés d'origine.

La banlieue difficile est devenue en France le lieu d'expression d'une jeunesse révoltée, particulièrement quand elle est d'origine maghrébine et africaine. Cette jeunesse est appelée à adhérer à un modèle républicain nonchalant en raison notamment de l'absence d'une intégration sociale par l'emploi, l'échec de la démocratisation de l'école et la persistance des discriminations. Confronté à l'embrasement des banlieues en 2005, l'État français a instauré un couvre-feu et des mesures essentiellement répressives. Or, la crise est profonde car le modèle républicain n'est plus capable de produire des références communes regroupant les différents groupes sociaux.

Dans ce tableau inquiétant sur la situation des banlieues françaises, il nous semble important de relever un apparent paradoxe scolaire français. Si les recherches suisses et Nord-américaines (abordées ultérieurement dans ce texte) montrent clairement une marginalisation scolaire des minorités ethniques, les études françaises montrent en revanche que les jeunes issus de familles immigrées font preuve d'un surcroît d'ambition scolaire (Caille, 2005). À caractéristiques sociodémographiques comparables, avoir des parents immigrés constitue, après l'origine sociale de l'élève, le déterminant le plus important du souhait de poursuite des études. Les chances d'entrer au lycée général sont de 20,7\% pour un enfant de famille non immigrée. À situation familiale et sociale identique, elles sont de 26,6\% pour un enfant dont la famille est originaire du Maghreb. Les jeunes issus des familles asiatiques ont plus de chances d'accéder au lycée comme le montre le tableau ci-dessous. 
Tableau 1: origine familiale et accès au lycée général

\begin{tabular}{|l|c|}
\hline enfants d'une famille non immigrée & $20,7 \%$ \\
\hline enfants d'une famille originaire du Maghreb & $26,6 \%$ \\
\hline enfants d'une famille originaire d'Asie du Sud-Est & $37,6 \%$ \\
\hline enfants d'une famille originaire d'Europe du Sud & $29,4 \%$ \\
\hline enfants d'une famille originaire de Turquie & $12,7 \%$ \\
\hline
\end{tabular}

Source: Baumard (2005)

Pour relativiser ce succès scolaire des jeunes issus de familles immigrés en France, il faut rappeler que la scolarisation n'a de sens que dans la mesure où elle anticipe une future insertion socioéconomique. Or, toutes les études convergent vers un même constat: à diplômes et qualifications scolaires équivalentes, un jeune issu de l'immigration maghrébine a beaucoup moins de chance qu'un français de souche de décrocher un emploi (Dupray \& Moullet, 2004; Frickey \& Primon, 2006). Par ailleurs, il faut noter que l'examen plus fin des filières scolaires que fréquentent les élèves du secondaire selon leurs origines ethniques montre une surreprésentation des élèves d'origine maghrébine dans les lycées professionnels. Or, les lycées professionnels, malgré leur désignation, ne permettent pas forcément une intégration par l'emploi et ne sont pas perçus par les jeunes qui les fréquentent comme professionnalisant (Charlot, 1999). Ajoutons qu'un tabou entoure en France la question des politiques d'accès à l'égalité pour les minorités ethniques (affirmative action). Longtemps, l'usage de ce concept d'origine anglosaxonne a buté sur un problème de traduction en français. En effet, la traduction de l'«affirmative action» par «discrimination positive» dans laquelle discrimination apparaît en premier donne au concept une connotation négative qui peut aller jusqu’à signifier le contraire de l'égalité.

Que faut-il retenir de cette analyse de la situation scolaire des minorités ethniques en France ? Les carrières scolaires des jeunes d'origine immigrés semblent comparables à celles des autres catégories de jeunes. Au-delà de cet effet paradoxal lié à la massification scolaire, il faut surtout retenir les faibles possibilités d'intégration par l'emploi et l'actualité des discriminations.

\section{Modèle multiculturel aux États-Unis: Racines historiques et retour de la ségrégation}

Revenons dans un premier temps sur les racines historiques du modèle multiculturel, ses réussites et ses échecs. L'éducation a été de tout temps en Amérique du Nord une institution liée aux pouvoirs politiques et religieux locaux et redevable à la communauté locale. Il s'agit là d'une tradition qui renvoie à la culture organisationnelle protestante des Etats-Unis depuis la première colonie de Jamestown. Ainsi, Kloss (1977) rapporte que 600000 élèves américains étaient scola- 
risés en allemand au début du 20 ème siècle. Historiquement le système scolaire des Etats-Unis se caractérise ainsi par une sensibilité forte aux particularismes locaux (linguistiques et religieux) et une ségrégation institutionnelle frappant les communautés noire, hispanique et amérindienne. C'est pourquoi les revendications des minorités ethniques à l'école prennent leur source dans les mouvements afro-américain, hispanique et indien de contestation de l'hégémonie et de la domination de la majorité WASP (blanche anglo-saxonne protestante) sur la forme scolaire. Cette contestation prend essentiellement la forme d'une lutte pour l'égalité et non pas la séparation des communautés ethniques. Ainsi, le droit de fréquenter une école de qualité, la même que les blancs, a longtemps été au centre des luttes du mouvement afro-américain pour les droits civiques. En 1954, la Cour suprême des Etats-Unis a déclaré inconstitutionnelle la ségrégation raciale dans les écoles publiques (Kluger, 1975). C'est le début de l'ouverture de l'école publique à la diversité culturelle aux États-Unis et une date majeure dans l'histoire du modèle multiculturel américain. Les mouvements hispanique et amérindien ont profité de cette brèche pour centrer leurs revendications sur les droits linguistiques et le contrôle des écoles qui servent leurs communautés. Avec la loi sur l'éducation bilingue (Bilingual Education Act) de 1968, l'État a reconnu, après des décennies de lutte, la possibilité d'un usage scolaire des langues autres que l'anglais (Ovando, 2003).

Favorisé par des mesures légales contraignantes (Affirmative Action, Bilingual Education Act), le paysage scolaire a été profondément chamboulé dans les années 60-70 aux États-Unis. Cependant, le bilan actuel semble mitigé. Selon l'étude de Frankenberg, Lee et Orfield (2003), un des indicateurs de la ségrégation scolaire est le contact entre élèves minoritaires et élèves blancs. Le pourcentage des élèves blancs dans les écoles noires a diminué de 5,3\% entre 1988 et 2000, retournant à un taux inférieur à ce qu'il était en 1970, avant le début du busing (transfert obligatoire en bus des élèves d'un quartier à un autre pour favoriser la mixité raciale). Le tableau 2 montre clairement que deux groupes ethniques sont placés dans des ghettos scolaires: les noirs et les hispaniques ${ }^{4}$.

Tableau 2: composition raciale typique de l'école fréquentée par différents groupes

\begin{tabular}{|l|c|c|c|c|c|}
\hline & $\begin{array}{c}\text { Elèves } \\
\text { blancs }\end{array}$ & $\begin{array}{c}\text { Elèves } \\
\text { noirs }\end{array}$ & $\begin{array}{c}\text { Elèves } \\
\text { hispaniques }\end{array}$ & $\begin{array}{c}\text { Elèves } \\
\text { asiatiques }\end{array}$ & $\begin{array}{c}\text { Elèves } \\
\text { indiens }\end{array}$ \\
\hline Blancs (\%) & 78 & 30 & 28 & 45 & 44 \\
\hline Noirs (\%) & 9 & 53 & 12 & 12 & 7 \\
\hline Hispaniques (\%) & 9 & 13 & 55 & 20 & 3 \\
\hline Asiatiques (\%) & 3 & 3 & 5 & 22 & 35 \\
\hline Indiens (\%) & 1 & 1 & 1 & 1 & 100 \\
\hline Total & 100 & 100 & 100 & 100 & 11 \\
\hline
\end{tabular}

Source: Frankenberg, E., Lee,C. \& Orfield, G. (2003) 
Les recherches ethnographiques d'Ogbu $(1987 ; 1982 ; 1994)$, poursuivies par Fordham (1996), montrent que la communauté afro-américaine est traversée par des conflits internes liées au positionnement des élèves noirs dans le système scolaire. La minorité d'élèves noirs qui réussit suscite un doute sur sa propre identité culturelle par la majorité marginalisée sur le plan scolaire. Autrement dit «réussir à l'école reviendrait à devenir blanc!». Paradoxalement, la fin légale de la ségrégation a diminué la cohésion ethnique de la communauté afro-américaine puisqu'on constate une fuite des classes moyennes et intellectuelles noires des ghettos vers les zones suburbaines mixtes ou blanches. La capacité de résistance culturelle de la communauté noire s'est ainsi trouvée largement amoindrie par ce découplage entre l'élite intellectuelle et les masses populaires. À titre d'exemple, dans une enquête réalisée à la fin des années 90 dans les quartiers noirs de Baltimore, aucun enseignant noir ayant participé à l'étude n'habite dans le quartier où il enseigne. Cela diminue son engagement pour l'amélioration de l'éducation locale et les opportunités de rencontres avec les parents (Akkari, Serpell, Baker \& Sonnenschein, 1998). Par ailleurs, il faut observer que les changements structurels dans le système éducatif dans son ensemble affectent aussi les conditions de scolarisation des jeunes issus des minorités ethniques. En effet, depuis au moins les années 1980, le système éducatif américain semble donner la priorité aux solutions néolibérales, privilégiant la concurrence entre établissements, le choix des écoles par les parents et le recours à l'enseignement privé. La persistance des difficultés qu'affrontent les minorités ethniques aux Etats-Unis s'est récemment illustrée par la contestation de deux mesures phares du modèle multiculturel: la politique d'accès à l'égalité (affirmative action) et l'éducation bilingue.

Par une politique préférentielle volontariste permettant l'accès à l'emploi et à l'enseignement supérieur de certaines catégories de population victimes d'une discrimination historique, les femmes et les minorités ethniques (noires, hispaniques, amérindiennes etc.), l'affirmative action a visé la réduction de la fracture entre la composition démographique du pays et la composition de son élite. De surcroît, l'éducation bilingue est depuis quelques années véritablement en état de siège dans de nombreux Etats. Le mouvement «English Only» militant contre les programmes d'éducation bilingue est monté en puissance dans l'ensemble du pays et en particulier en Californie qui abrite une importante communauté hispanophone. De même, une récente décision de la cour suprême contre l'action affirmative a semé le doute sur la légalité de cette mesure.

L'essoufflement du modèle multiculturel a été mis en évidence par Ogbu (1992) qui estime que les réformes du curriculum scolaire par l'éducation multiculturelle se sont avérées inadéquates pour lutter contre les inégalités, notamment parce que ces approches ignorent les conflits identitaires face aux apprentissages scolaires. Ces derniers sont pour les cultures minoritaires connotés en termes de domination et d'oppression.

The crucial issue in cultural diversity and learning is the relationship between the minority cultures and the American mainstream culture. Minorities whose cultu- 
ral frames of reference are oppositional to the cultural frame of reference of American mainstream culture have greater difficulty crossing cultural boundaries at school to learn (p. 5).

Par ailleurs, les réformes du curriculum scolaire tentant de valoriser les cultures minoritaires dans la classe et de réduire les stéréotypes sont souvent inefficaces car elles n'encouragent pas les élèves minoritaires à assumer la pleine responsabilité pour leurs apprentissages scolaires. De plus, ces réformes ne se basent pas sur une connaissance approfondie des cultures minoritaires, en tant que constructions dynamiques et complexes. Loin d'être de nature essentialiste comme le postulent certaines approches multiculturelles dominantes ${ }^{5}$, les cultures minoritaires sont traversées par des conflits et font l'objet de transformations permanentes. En définitive, la revendication multiculturelle aux USA est issue d'un mouvement historique pour l'égalité devant la forme scolaire. Le bilan que l'on peut aujourd'hui tirer de cette expérience est mitigé. La ségrégation ne s'est jamais arrêtée et reprend de l'ampleur, les quelques gains légaux obtenus par les minorités ethniques sont souvent remis en question.

\section{Cultures et scolarisation en Suisse: Le poids des stéréotypes}

L'extrême décentralisation du système éducatif suisse limite la possibilité de faire une analyse similaire à celles que nous avons effectuées dans le cas de la France et des États-Unis. En effet, une analyse de la situation scolaire des minorités ethniques doit toujours se faire au niveau cantonal en Suisse. Cependant, nous pouvons dire que les modèles adoptés dans les approches de la scolarisation des élèves migrants ou d'origine étrangère ${ }^{6}$ en Suisse puisent leur inspiration dans quatre sources principales. Tout d'abord, une conception ethnique de la nation dans laquelle on acquiert l'identité nationale par le droit du sang. Cette source partagée avec l'Allemagne guide la manière dont on pense la scolarisation des élèves migrants ou d'origine étrangère et leurs différences culturelles. La deuxième source, principalement présente dans les cantons romands, puise dans les référentiels républicains français avec une mise en avant de la laïcité, de l'égalité et du mérite. Elle est particulièrement prégnante dans les cantons de GE, JU et NE. La troisième source, visible principalement dans les grandes villes, assume la multiculturalité de fait de la société suisse actuelle et utilise les sources du cosmopolitisme et du multiculturalisme. La quatrième source découle de la politique des trois cercles qui qui a tracé au début des années 90 les grandes lignes de la politique migratoire suisse excluant ou réduisant l'apport migratoire en provenance des pays du Sud (Conseil Fédéral, 1991).

Ces quatre sources, parfois en tension, se traduisent obligatoirement en matière de scolarisation des élèves d'origine étrangère. C'est tout d'abord la surreprésentation des élèves de nationalité étrangère dans les filières scolaires dévalorisées qui retient notre attention. En Suisse, de nombreuses recherches tendent à montrer la surreprésentation des enfants étrangers dans les sections scolaires les moins exi- 
geantes $^{7}$ (Lischer, 1997; Akkari \& Gohard-Radenkovic, 2002). Ce constat rend souvent «l'école multiculturelle» synonyme d'école à faibles exigences ou d'école à problèmes, notamment auprès de certains parents et enseignants. Dans les établissements scolaires, Lanfranchi (2002) montre que les enfants de familles immigrées sont souvent jugés moins aptes à la scolarisation que les enfants suisses. Les enfants issus d'un milieu d'immigrés sont également surreprésentés par rapport à leurs camarades suisses parmi les élèves qui redoublent. A cet égard, l'orientation vers les classes spéciales des enfants étrangers est une question cruciale. Dans les cantons de Zoug, de Schaffhouse et d'Argovie, un enfant étranger sur six fréquente une classe spéciale ${ }^{8}$; ce pourcentage est largement supérieur à leur proportion dans les classes ordinaires (Lischer, 2003; Coradi Vellacott \& Wolter, 2005). Il est pertinent de remarquer que l'encadrement spécial ou de «retrait» des élèves du circuit normal n'est pas répandu de manière égale dans tous les cantons. Selon Lischer (2004), 4,2\% des élèves de toute la Suisse sont formés au sein d'une classe spéciale en 2003. Cette proportion varie entre 1,2\% dans le canton du Valais et 7,0\% dans celui d'Argovie. La part des élèves dans les classes spéciales a énormément augmenté depuis 1980. Cette hausse s'explique essentiellement par une prise en charge croissante d'élèves d'origine étrangère. Un enfant étranger sur 11 est scolarisé dans une classe spéciale, ce qui n’est le cas que d'un enfant suisse sur 40 .

Dans les enquêtes PISA, la Suisse se distingue par le contraste apparent entre les résultats des jeunes issus de familles suisses et immigrées. Le tableau 3 montre que les enfants provenant de familles suisses ont des performances scolaires beaucoup plus élevées que les enfants issus de familles immigrés.

Tableau 3: influence de l'origine sociale et de la taille de la famille sur les performances scolaires ${ }^{*}$

\begin{tabular}{|l|c|c|}
\hline & Familles suisses & Familles immigrés \\
\hline Famille de 2 enfants, statut social élevé & 569 & 492 \\
\hline Famille de 2 enfants, statut social inférieur & 484 & 443 \\
\hline Famille de 5 enfants statut social inférieur & 475 & 379 \\
\hline
\end{tabular}

*L'échelle PISA des compétences en lecture a six niveaux qui s'échelonnent de 335 à 626 points. La valeur moyenne suisse est de 494

Source: Wolter \& Coradi Vellacott (2002). 
Les élèves issus de familles immigrées, en particulier de statut socioéconomique peu élevé, sont aussi désavantagés lors du passage du primaire au secondaire. L'étude de Haeberlin, Imdorf \& Kronig (2004) portant sur un échantillon de 1'367 élèves de sixième année en Suisse alémanique a mis en évidence que les garçons de familles immigrées et présentant un niveau moyen de compétences font l'objet d'une nette discrimination. Les auteurs soulignent qu'une jeune fille suisse ayant obtenu des résultats scolaires moyens a $83 \%$ de chances d'être orientée vers une classe de la filière à exigences élevées (plutôt que vers une classe de la filière à exigences élémentaires), tandis qu'un garçon issu d'une famille immigrée et présentant strictement les mêmes résultats scolaires n'a que 37\% de chances de bénéficier de la même orientation.

S’il est difficile dans le cadre de cet article d'approfondir toutes les raisons qui amènent le système scolaire suisse à ne pas traiter les élèves d'origine étrangère d'une manière équitable, on peut penser que les représentations et les stéréotypes que les enseignants en formation ou en activité véhiculent à propos des élèves d'origine étrangère expliquent une partie du problème. Comme l'a mis en évidence Gremion (2005), il suffit de peu d'indices suggérant une différence culturelle ou une altérité pour que les stéréotypes se mettent à l'œuvre.

Dans le cadre d'une enquête récente inspirée de la recherche de Chryssochoou, Picard et Pronine (1998), nous avons analysé les représentations particulières que 45 enseignants romands véhiculent à propos d'élèves supposés être d'origine ethnique ou culturelle différente? ${ }^{9}$. Nous avons opté pour un indicateur construit à partir du prénom des élèves. Ce dernier peut suggérer à l'acteur scolaire une origine culturelle potentiellement différente. Les participants à notre enquête étaient confrontés à l'analyse du cas d'une jeune élève de 13 ans ayant toujours vécu en Suisse romande. Cette élève qui fréquente la deuxième année de l'enseignement secondaire se trouve en grande difficulté scolaire. Son bilan scolaire est préoccupant puisque la plupart de ses notes sont inférieures à la moyenne notamment en mathématiques et en français. Des informations sur la situation familiale étaient également fournies. L'élève est née en Suisse, ses parents vivent ensemble, sa mère n'a pas d'activité professionnelle, elle a un frère de 8 ans. La moitié des enseignants avaient analysé le cas de Céline, et l'autre moitié le cas de Naïma, une autre élève. Les parents de Céline étaient présentés comme suisses et ceux de Naïma étaient présentés comme d'origine étrangère.

En dépit d'un dossier scolaire strictement équivalent, le discours des enseignants romands pour analyser et proposer des solutions pédagogiques pertinentes aux deux cas analysés était clairement lié au prénom auquel ils étaient confrontés. Les problèmes scolaires de Céline étaient attribués à l'étape difficile de l'adolescence et aux tensions au sein de sa famille. Les enseignants se sont montrés toutefois assez confiants quant à son avenir scolaire. Naïma, par contre, était perçue comme victime d'un conflit de loyauté lié à l'origine étrangère de ses parents (assise entre 2 chaises !). Le statut de la femme dans son pays d'origine serait très défavorable à son succès scolaire et la probabilité que les parents ne par- 
lent pas français entre eux à domicile serait une des explications de son échec scolaire. Les enseignants se sont montrés beaucoup plus réservés quant à son avenir scolaire comparativement à Céline. Dans l'ensemble, cette recherche nous incite à penser que si l'analyse par les enseignants de l'échec scolaire d'un élève peut tenir en partie au prénom, leur attitude générale envers lui aussi ! (Akkari, sous presse). La théorie de la reproduction a clairement montré que l'école transforme les différences socioculturelles en différences de statut scolaire. Parmi ces différences ainsi transposées, il y a les différences ethniques: l'école peut transformer les différences ethniques qui existent dans son contexte en différences scolaires. Et ces dernières peuvent en retour avoir des effets sur les relations ethniques dans l'environnement social de l'école (Lorcerie, 2006).

\section{Cultures oppositionnelles et forme scolaire}

Dans cette dernière partie de l'article, nous souhaitons faire la synthèse de l'examen de la scolarisation des minorités ethniques dans les trois contextes nationaux abordés. Même si elle a été forgée principalement aux États-Unis, il nous semble que l'approche de l'anthropologue de l'éducation Ogbu (cultural-ecological theory of minority academic achievement) permet de saisir les enjeux de la scolarisation des minorités ethniques dans les trois pays. Ogbu affirme d'abord que l'école est un milieu ouvert à la société et que le traitement des minorités ethniques dans la société se reflète automatiquement dans leur traitement à l'école. Ogbu (1992) suggère que les minorités involontaires ont un cadre de référence qui n'est pas seulement différent de celui dominant à l'école mais probablement en opposition. Le cadre d'analyse d'Ogbu se base sur la séparation des minorités ethniques en deux groupes: les immigrants (minorités volontaires) et les minorités involontaires qui peuvent être considérées comme des castes inférieures. Dans le cas des États-Unis, Ogbu désigne comme minorités involontaires les personnes qui ont été amenées dans le giron des États-Unis par l'esclavagisme, la conquête territoriale et le travail forcé. Alors que les minorités volontaires s'assimilent rapidement à l'école américaine en contournant les obstacles de départ qui entravent leur scolarisation, les minorités involontaires considèrent l'école comme équivalent de l'apprentissage de la culture et de la langue des Américains blancs, c'est-à-dire l'apprentissage du cadre de référence culturel de leurs oppresseurs historiques.

La théorie d'Ogbu doit être adaptée et reformulée afin de pouvoir s'appliquer à la situation scolaire des jeunes issus des minorités ethniques en France ou en Suisse. Nous proposons une troisième catégorie intermédiaire entre les minorités volontaires (immigrants) et les minorités involontaires (castes inférieures): les minorités postcoloniales ${ }^{10}$. Le rapport postcolonial recouvre deux dimensions principales: la dépendance économique ou culturelle et la menace perçue par la majorité de la présence durable d'une minorité ethnique. Si on examine l'application de la théorie d'Ogbu dans le cas de la France, on constate que les pays 
d'origine vivent une dépendance économique et culturelle importante par rapport à la France (échanges commerciaux, aide au développement, flux migratoires etc....) même si la plupart des immigrés maghrébins ou africains sont venus volontairement en France. Au niveau culturel, il suffit de mentionner l'usage du français comme langue principale d'enseignement en Afrique du Nord et de l'Ouest. La deuxième dimension du rapport postcolonial concerne la perception par la majorité autochtone de la présence durable d'une minorité ethnique comme menaçante. C'est ce qu'on peut appeler la construction sociale de certains groupes ethniques comme "autres différents». Une sorte d'altérité culturelle radicale se met à l'oeuvre: «Il y a des gens comme nous ......Mais, ceux là ne sont pas comme nous et ne le seront probablement jamais» ${ }^{11}$. Ce processus d'ethnicisation absolue désigne le fait d'imputer une origine aux individus, ou d'en revendiquer une, de telle sorte que cette croyance influence l'action sociale et structure le monde d'une manière duale et hermétique entre un «nous ou ceux qui sont comme nous» et «eux ou les autres». L'ethnicisation varie selon les contextes et peut se baser sur la race, la langue, la religion, le mode vie, la culture ou toute autre caractéristique saillante. Ainsi en France, c'est essentiellement les jeunes d'origine maghrébine et africaine qui font l'objet d'une ethnicisation des rapports sociaux à l'école. En arrière plan, c'est la religion et la couleur qui sont en cause. Aux États-Unis, ce sont les jeunes noirs et hispaniques qui expriment des attitudes oppositionnelles à l'école. En Suisse, ce sont essentiellement les jeunes originaires du Kosovo, de Bosnie ou de Turquie et ceux des autres régions du Sud ${ }^{12}$ qui font l'objet d'un débat tranché en termes de «nous» et "eux». Même si la Suisse n'a pas de passé colonial, elle est fortement impliquée dans des échanges économiques internationaux inégaux avec la plupart des pays du Sud.

Il faut observer que la transmission de la catégorisation «ne sont pas comme nous» n'est pas forcément liée aux conditions d'existence ou aux comportements réels de la population ethnique cible. Ainsi, à de nombreuses reprises, aussi bien en Suisse qu'en France, des enseignants peuvent utiliser la catégorisation "...ne sont pas comme nous» pour des jeunes nés dans la même ville qu'eux, ayant fréquenté les mêmes écoles, parlant la même langue et vibrant pour les mêmes clubs de football. Cette construction ethnique de l'altérité à l'école n'est pas sans liens avec le développement d'un discours raciste général dans la société (et pas uniquement au sein des partis extrémistes de droite) qui allie l'encensement nostalgique de l'homogénéité culturelle positive d'autrefois (la Suisse et la France ne sont plus ce qu'elles étaient !) et une peur constante des conflits culturels amenés par les migrations.

En définitive, il nous semble important de noter que même si les attitudes oppositionnelles et conformistes ne sont pas les seules options possibles face à la forme scolaire, les jeunes appartenant à des minorités involontaires et postcoloniales représentent une problématique prioritaire si l'on veut impulser plus d'équité au niveau scolaire et social. Suite à la massification scolaire, l'équité doit se traduire dans la qualité des apprentissages et des trajectoires scolaires. 


\section{La perspective de futures recherches}

Cette analyse des impasses de la forme scolaire dans différents contextes nationaux nous incite à proposer des priorités de recherche dans ce domaine pour les prochaines années. Si le modèle républicain à la française a fait la preuve de son échec manifeste notamment dans les banlieues, le modèle multiculturaliste à l'anglo-saxonne n'a pas non plus réussi à enrayer la ségrégation raciale à l'école. En Suisse, l'origine étrangère signifie souvent des trajectoires scolaires à moindres exigences. Quel que soit le contexte scolaire étudié, nous avons constaté que la forme scolaire n'offre pas aux jeunes appartenant à des minorités ethniques les mêmes opportunités qu'au reste des jeunes. Non seulement, ces jeunes sont souvent identifiés a priori comme une population scolaire à problèmes (à risques), parfois (voire souvent) à tort, mais certains jeunes minoritaires n'identifient pas la réussite scolaire comme un comportement social désirable. Les jeunes appartenant à des minorités «involontaires» et "postcoloniales» opposent à la forme scolaire des identités ethniques recomposées, ont de la peine à avoir des relations intergénérationnelles constructives au sein de leurs propres communautés et tombent parfois dans des conduites délinquantes. Il nous semble utile de poursuivre les recherches afin de comprendre les raisons de ce divorce très risqué pour l'avenir des sociétés contemporaines. Nous voulons proposer ci-dessous quelques pistes susceptibles d'impulser une meilleure connaissance de cette problématique.

En premier lieu, la recherche doit porter une attention particulière à la tranche d'âge 12-15 ans des jeunes appartenant à des minorités ethniques. Etant donnée sa complexité et son importance dans la structuration de la personnalité d'un adulte, cette période de la vie serait celle qui donnerait aux chercheurs le plus de possibilités d'analyser les mécanismes de l'attitude scolaire oppositionnelle. En second lieu, il nous semble utile de poursuivre les études sur la manière dont les enseignants appréhendent et gèrent les différences culturelles dans leurs classes. Au-delà de leur discours récurrent sur l'égalité ou l'indifférence aux différences culturelles, il serait intéressant d'analyser les jugements qu'ils portent sur leurs élèves en fonction de l'appartenance ethnique (culturelle) réelle ou perçue. Il serait également essentiel d'étudier l'efficacité des actions de formation initiale et continue en approches interculturelles. En effet, il nous paraît important d'évaluer l'impact des différents programmes de formation à l'interculturel et d'identifier les activités qui font la différence en matière de sensibilité accrue à la diversité culturelle. En troisième lieu, il sera nécessaire d'examiner les pratiques concrètes de gestion des différences culturelles par le système scolaire, notamment par le biais de l'observation des mécanismes en œuvre dans les procédures de composition des classes, d'orientation et de placement des élèves.

En conclusion, la situation scolaire des jeunes appartenant à des minorités ethniques est un enjeu de taille pour les systèmes scolaires urbains dans les trois contextes abordés dans cet article. La capacité de la forme scolaire de se rénover 
pour répondre aux besoins spécifiques de ces élèves sera au centre des politiques éducatives pour les prochaines années. Comme le souligne à juste titre Castagny (2003, p. 28), la formule souvent entendue «ils n'ont qu'à s'adapter» fait l'économie d'une réelle explication des droits et des devoirs de chacun, dans les établissements scolaires et dans la société en général: «La dimension affective et psychologique dans cette adaptation est laissée à la seule charge de l'élève d'origine étrangère». Il serait utile d'identifier les moyens d'amener aussi bien les enseignants que les élèves issus des minorités ethniques à séparer le succès scolaire de l'obligation d'une assimilation totale au modèle dominant («agir comme un blanc» Acting White) ou selon l'expression de Ogbu (1992, p. 12) «à Rome, fais comme les Romains sans devenir Romain». Enfin, la société dans son ensemble doit s'interroger non seulement sur les discriminations multiples frappant les minorités, en particulier en matière d'emploi ou d'éducation, mais aussi sur les principes qui doivent guider la politique migratoire et les échanges économiques et culturels avec les pays du Sud.

\section{Notes}

1 De nombreux travaux francophones ont conceptualisé la notion d'ethnicité (Lorcerie, 2003, 2006; Guillaumin, 2002). Le concept d'ethnicité concerne la façon dont l'origine supposée des individus influence l'interaction sociale. Différentes catégorisations sont utilisées dans les trois contextes nationaux abordés dans cet article. Aux Etats-Unis, les catégories raciales et ethniques sont officiellement utilisées par l'administration publique et les institutions éducatives. En revanche, en France, l'utilisation des catégories ethniques est peu courante en dehors de certains travaux de recherche. Des expressions indirectes telles que français d'origine maghrébine ou africaine sont les plus fréquemment utilisées. En Suisse, la catégorie «étrangers» et d'origine étrangère sont les plus utilisées.

2 Rappelons que ce modèle s'enracine dans l'époque de Jules Ferry marquée à la fois par la consolidation de l'école publique laïque mais aussi par les conquêtes coloniales.

3 En France, l'enseignement privé, essentiellement catholique, scolarise chaque année environ $17 \%$ des élèves tous niveaux confondus (Chauffaut, Olm \& Simon, 2005).

4 Malgré la reconnaissance politique de la communauté hispanique et un accroissement de son poids démographique et économique, les jeunes appartenant à cette communauté constituent le groupe ethnique le plus marginalisé sur le plan scolaire. La ségrégation raciale accompagne toujours la ségrégation due à la pauvreté et à bien d'autres formes d'inégalités sociales. Les niveaux de compétition scolaire entre élèves et l'appui des parents sont beaucoup plus faibles dans les écoles avec peu de ressources, c'est-à-dire essentiellement celles qui desservent les minorités ethniques. Les enseignants qualifiés et expérimentés se détournent souvent de telles écoles.

5 Malgré leur apport incontournable à l'éducation interculturelle, deux des principaux livres utilisés aux Etats-Unis (Banks \& Banks, 1993; Bennett; 1995) montrent les difficultés de se distancer d'une approche figée et essentialiste des cultures dans l'espace scolaire. Peu de chapitres dans ces deux ouvrages édités à de nombreuses reprises laissent de la place aux identités hybrides et aux métissages.

6 Pour analyser la problématique de la diversité culturelle et sa gestion, la plupart des études utilisent en Suisse la terminologie «élèves immigrés/migrants» ou "élèves étrangers». Toutefois, cette terminologie ne correspond pas forcément à ce que les acteurs du terrain scolaire considèrent comme «diversité ou différence culturelle». En effet, même possédant la nationalité suisse, certains élèves dont les parents sont d'origine étrangère sont considérés 
comme différents et font l'objet de représentations et de comportements en terme de distance culturelle.

7 Quelque soit l'effort consenti en matière de terminologie pour réduire la connotation négative de ces sections (classe de développement, section générale etc....), la plupart des observateurs constatent que les élèves orientés vers ces sections ont peu de possibilité de poursuivre leurs études.

8 Aux États-Unis ce problème a été identifié il y a près de 38 ans et est resté largement ignoré par les chercheurs et les praticiens (Artiles, Klingner \& Tate, 2006)

9 A ce propos, la nationalité des élèves utilisée dans la plupart des recherches est une variable insuffisante pour mesurer la ségrégation ethnique. En effet, la question de la nationalité, dimension essentiellement juridique et politique, ne recouvre que partiellement celles de la ségrégation et de la discrimination.

10 Le rapport postcolonial ne dépend pas seulement de l'existence d'un contentieux colonial entre 2 peuples, mais aussi de la nature des rapports économiques, politiques et culturels qu'ils entretiennent actuellement. Ainsi, même si la Suisse n'a pas eu des colonies à l'instar d'autres Etats européens, elle maintient un rapport de domination de type postcolonial avec les pays du Sud. La Direction du développement et de la coopération (2007) a récemment mis en évidence le fait que l'Afrique finance la Suisse dans la mesure où les investisseurs suisses retirent de ce continent plus d'argent qu'ils n'y réinvestissent.

11 Certains auteurs principalement anglo-saxons ont conceptualisé la stigmatisation et la ségrégation dont font l'objet certaines minorités ethniques par le concept de "Whiteness» (blancheur), difficilement traduisible en français mais qui renvoie à la fois à l'eurocentrisme et au privilège d'être blanc (Sleeter, 2001). Il nous semble que l'usage que certains font de l'identité européenne (blanche et chrétienne) se rapproche de ce privilège.

12 Tout se passe comme si des anciens immigrés (italiens, espagnols, voire portugais) deviennent de bons immigrés, de surcroît issus des pays de l'Union Européenne et sont remplacés par les plus récents dans les représentations négatives (Balkans, Turcs et ceux originaires des pays du Sud). Il faut remarquer que presque tous les jeunes originaires d'Europe du Nord suivaient une formation post-obligatoire, la proportion des Espagnols et Italiens était de deux tiers, alors que seul un tiers des ex-Yougoslaves, Turcs et Portugais se trouvaient dans ce type de formation (Lischer 1997). Il faut toutefois rappeler que l'intégration dans la société d'accueil est à considérer, dans une cadre global qui inclut les trajectoires scolaires mais aussi l'insertion par l'emploi. Or, les portugais compensent leur sortie plus précoce du système scolaire par rapport aux autres migrants en provenance de l'Union Européenne par une meilleure intégration professionnelle par rapport aux migrants originaires de l'ex-Yougoslavie (Fibbi, Kaya \& Piguet, 2003). La même tendance a été constatée en France dans la comparaison de l'insertion des jeunes d'origine portugaise et maghrébine (Okba \& Lainé, 2005).

\section{Références}

Akkari, A. (sous presse). Représentations, pratiques pédagogiques et traitement de la différence socioculturelle dans le système scolaire. In C. Perregaux (Éd.), Les approches interculturelles et interlangagières entre savoirs issus de la recherche et savoirs d'expérience: une interculturalité nécessaire ? Paris: L'Harmattan.

Akkari, A., Serpell, R., Baker, L. \& Sonnenschein, S. (1998). A comparative analysis of teacher ethnotheories. Professional Educator, 21 (1), 45-59.

Akkari. A. \& Gohard-Radenkovic, A. (2002). Vers une nouvelle culture pédagogique dans les classes multiculturelles: les préalables nécessaires. Revue suisse des sciences de l'éducation, 18 (1), 147-170.

Artiles, A.J., Klingner, J. K. \& Tate, W. F. (2006). Representation of minority students in special education: complicating traditional explanations. Educational Researcher, 35 (6), 3-5. 
Banks, J. A., \& Banks, C .A. M. (Éd.). (1993). Multicultural education: Issues and perspectives (2nd ed.). Boston: Allyn and Bacon.

Baumard, M. (2005). Lycées: fils d'immigrés et motivés. Le Monde de l'Education, 5, 56- 57.

Benbassa, E. \& Attias, J.-C. (2007). Des cultures et des dieux. Repères pour une transmission du fait religieux. Paris: Fayard.

Bennett, C. I. (1995). Comprehensive multicultural education theory and practice (3rd ed.). Boston: Allyn and Bacon.

Bourdieu, P. (1991). L'ordre des choses. Entretiens avec des jeunes gens du nord de la France. Actes de recherches en Sciences Sociales, 90, 7-19.

Bourdieu, P. (1993). La misère du monde. Paris: Seuil.

Bourdieu, P. \& Passeron, J.-C. (1970). La Reproduction: éléments d'une théorie du système d'enseignement. Paris: Éd. de Minuit.

Caille, J.-P. (2005). Les projets d'avenir des enfants d'immigrés. Paris: DEP-Ministère de l'éducation.

Castany, J. (2005). L’intégration au «quotidien». Les Cahiers Pédagogiques, 419, $27-28$.

Charlot, B. (1999). Le rapport au savoir en milieu populaire. Paris: Anthropos.

Chauffaut, D., Olm, C. \& Simon, M.-O. (2005). L'enseignement libre: choix de conviction mais aussi de pragmatisme. Paris: Centre de recherche pour l'étude et l'observation des conditions de vie.

Chryssochoou, X., Picard, M., \& Pronine, M. (1998). Explications de l'échec scolaire. Les théories implicites des enseignants selon l'origine sociale et culturelle de l'élève. Psychologie \& Éducation, 32, 43-59.

Conseil Fédéral (1991). Rapport du Conseil Fédéral sur la politique à l'égard des étrangers et des réfugiés du 15 mai 1991. Berne: Conseil Fédéral (no. 91.036).

Coradi Vellacott, M. \& Wolter, S. (2005). L'égalité des chances dans le système éducatif suisse. Aarau: Centre suisse de coordination pour la recherche en éducation.

Direction de la coopération et du développement. (2007). Les interactions de la Suisse avec le monde. Berne: Direction de la coopération et du développement.

Dupray, A. \& Moullet, S. (2004). Linsertion des jeunes d'origine maghrébine en France. Des différences plus marquées dans l'accès à l'emploi qu'en matière salariale. Marseille: Céreq.

Fibbi, R., Kaya, B. \& Piguet, E. (2003). Afrim ou Mehmet fait la différence. Berne: Fonds national suisse de la recherche scientifique.

Fordham, S. (1996). Blacked out: Dilemmas of race, identity, and success at capital high. Chicago: University of Chicago Press.

Frankenberg, E., Lee, C. \& Orfield, G. (2003). A multiracial society with segregated schools: Are we losing the dream? Cambridge, MA: The Civil Rights Project-Harvard University.

Frickey, A. \& Primon, J.-L. (2006). Insertion professionnelle et discriminations: l'accès à l'emploi des étudiants issus de l'immigration en région Provence - Alpes - Côte d'Azur, Cahiers de l'Urmis, mis en ligne le 11 décembre 2006:

[http://urmis.revues.org/document251.html]. [Consulté le 30 septembre 2007].

Gremion, L. (2005). Apprendre à voir l'Autre: pour une formation des enseignants à l'accueil de la diversité. Formation et pratiques d'enseignement en questions, 4, 13-34.

Guillaumin, C. (2002). L’idéologie raciste, genèse et langage actuel. Paris: Gallimard.

Haeberlin, U., Imdorf, Ch. \& Kronig, W. (2004). Von der Schule in die Berufslehre. Untersuchungen zur Benachteiligung von ausländischen und von weiblichen Jugendlichen bei der Lehrstellensuche. Bern: Haupt.

Jamet, C. Lenoir, Y. \& Xypas, C. (2006). (Éd.). Ecole et citoyenneté: un défi multiculturel. Paris: Armand Colin.

Kintzler, C. (1998). Tolérance et lä̈cité. Nantes: Editions Pleins Feux.

Kloss, H. (1977). The American bilingual tradition. Rowley, MA: Newbury House.

Kluger, R. (1975). Simple justice: The history of brown v. board of education and black america's struggle for equality. New York: Vintage Books. 
Lanfranchi, A. (2002). Schulerfolg von Migrationskindern. Die Bedeutung familienergänzender Betreuung im Vorschulalter. Opladen: Leske + Budrich.

Lischer, R. (1997). Intégration: une histoire d'échec ? Les enfants et les adolescents étrangers face au système suisse de formation. Berne: Office fédéral de la statistique.

Lischer, R. (2003). Intégration réussie des étrangers? La réponse des statistiques. Les enfants et adolescents étrangers dans le système suisse d'éducation et de formation. In CDIP, Le parcours scolaire et de formation des élèves immigrés à "faibles" performances scolaires. Convegno 2002, Rapport final. Berne: CDIP.

Lischer, R. (2004). Le système d'éducation suisse: une sélection d'indicateurs. Neuchâtel: Office fédéral de la statistique.

Lorcerie, F. (2003). L'effet «outsider». En quoi l'ethnicité est un défi pour l'école ? Ville-ÉcoleIntégration Enjeux, 135, 86-102.

Lorcerie, F. (2006). Quelle politique scolaire contre la catégorisation ethnique ? Le Télémaque, 29, 43-56.

Ogbu, J. U. (1994). Racial stratification and education in the United States: Why inequality persists. Teachers College Record, 96, 264-298. Retrieved August 2, 2002 from: [www.tcrecord.org].

Ogbu, J. U. (1982). Cultural discontinuities and schooling. Anthropology and Education Quarterly, 13 (4), 290-307.

Ogbu, J. U. (1987). Ethnoecology of urban schooling. New York: Colombia University Press.

Ogbu, J. U. (1992). Understanding cultural diversity and learning. Educational Researcher, 21(8) 5-14.

Okba, M. \& Lainé, F. (2005). L'insertion des jeunes issus de l'immigration: de l'école au métier. Paris: Conseil de l'Emploi, des Revenus et de la Cohésion sociale (CERC).

Ovando, C. J. (2003). Bilingual education in the United States: Historical development and current issues. Bilingual Research Journal, 27(1), 1-24.

Maurin, E. (2004). Le ghetto français. Paris: Seuil.

Ruano-Borbalan, J.C. (2002). L'école, ça sert d'abord à faire la société. Sciences Humaines, 36, 46-49.

Sleeter, C. E. (2001). Preparing teachers for culturally diverse schools: research and the overwhelming presence of whiteness. Journal of Teacher Education, 52, 94-106.

Vincent, G. (Éd.). (1994). L'éducation prisonnière de la forme scolaire? Scolarisation et socialisation dans les sociétés industrielles. Lyon: Presses Universitaires de Lyon.

Wolter, S. C. \& Coradi Vellacott, M. (2002). Sibling rivalry: A look at Switzerland with PISA data. IZA Discussion Paper No. 594.

Mots clés: culture, politiques éducatives, minorités ethniques, formation des enseignants, discrimination, inégalité. 


\section{Eine vergleichende Perspektive der multikulturellen und der republikanischen Schule}

\section{Zusammenfassung}

Dieser Text analysiert in einer vergleichenden Perspektive die Herausforderung, die das Wachstum der kulturellen Vielfalt in den Bildungssystemen der Industrieländer bedeutet. Im ersten Teil des Textes kommen wir auf den historischen Streit zwischen Schulform und bestimmten gesellschaftlichen Gruppen zurück. Der zweite Teil befasst sich mit der Analyse der Persistenz ungleicher Behandlung von ethnischen Minderheiten in verschiedenen Schulsystemen. Gegenstand des dritten Teils ist der Begriff der postkolonialen Minderheit als eine Bereicherung des von dem Anthropologen John Ogbu verwendeten Ansatzes in seiner Analyse der Schullaufbahnen von aus Minderheiten stammenden Jugendlichen. Im letzten Teil werden wir auf die Perspektive zukünftiger Forschungen eingehen, die den Schulen erlauben soll, kulturellen Unterschieden gegenüber sensibler zu werden.

Schlagworte: Kultur, Bildungspolitik, Minderheit, Lehrer/innenbildung, Ungleichheit

\section{Una visione comparativa sulla scuola multiculturale}

\section{Riassunto}

Il contributo analizza con un'ottica comparativa la sfida rappresentata dall'incremento della diversità culturale nei sistemi educativi dei paesi industrializzati. Nella prima parte affrontiamo il contenzioso storico legato al rapporto tra scuola e gruppi sociali, mentre nella seconda si analizza la persistenza del trattamento iniquo delle minoranze etniche nei differenti sistemi scolastici. La terza parte propone la nozione di minoranza postcoloniale quale arricchimento dell'approccio utilizzato dall'antropologo John Ogbu per analizzare i percorsi dei giovani provenienti da una minoranza. L'articolo si conclude con la discussione delle esigenze di ricerca in rapporto alla prospettiva di permettere alla scuola una maggiore sensibilità nei confronti delle differenze culturali.

Parole chiave: cultura, politiche educative, minoranze etniche, discriminazione, formazione degli insegnanti, disparità 


\section{Dealing with cultural diversity in schools in a comparative perspective}

\section{Summary}

Using a comparative perspective, this text analyzes the challenges that increasing ethno-cultural diversity present to educational systems in industrialized countries. In the first section we look back at the contentious history between formal schooling and certain social groups. The second part presents an analysis of the persistence of unequal treatment of ethnic minorities in different school systems. The third part point out the need to introduce the term "post-colonial minority" to enhance Ogbu's theory on Cultural discontinuities and schooling. In the final section we discuss future research perspectives that may allow formal schooling to become more sensitive to cultural differences.

Key words: culture, politiques éducatives, minorités ethniques, formation des enseignants, discrimination, inégalité 